\title{
Effect of Neoadjuvant Hormonal Treatment on the Necessity of Secondary Radiotherapy in Patients Undergoing Radical Prostatectomy for High-Risk Prostate Cancer
}

\author{
(1) Csaba Berczi ${ }^{1}$, (1) Janos Docs ${ }^{1}$, (1) Ben Thomas², (1) Zsolt Bacso ${ }^{3}$, (1) Tibor Flasko ${ }^{1}$ \\ ${ }^{1}$ Department of Urology, University of Debrecen, Debrecen, Hungary \\ 2Department of Internal Medicine, University of Debrecen, Debrecen, Hungary \\ ${ }^{3}$ Department of Biophysics and Cell Biology, University of Debrecen, Debrecen, Hungary
}

\begin{abstract}
Objective: The goal of this study was to see how neoadjuvant hormonal therapy affected the frequency of secondary radiotherapy after radical prostatectomy for high-risk prostate tumors.

Materials and Methods: Further, 527 patients with high-risk prostate cancer were divided into two groups. In group 1 (n=139), neoadjuvant androgen deprivation treatment was administered prior to surgery, whereas it was not applied preoperatively in group $2(n=388)$.

Results: Biochemical progression was observed in 27 patients (19.4\%) in group 1 and 54 patients (13.9\%) in group 2 ( $p=0.148$ ). Adjuvant and salvage irradiation were administered to patients with pT2 cancer who received neoadjuvant hvsormonal therapy in $17.3 \%$ and $7.1 \%$ of the cases, respectively, whereas in cases without prior neoadjuvant treatment, adjuvant and salvage irradiation were administered in $21.6 \%$ and $5.4 \%$ ( $p=0.370$ and $p=0.523$ ). Clinically advanced cancer patients who received neoadjuvant hormonal treatment had adjuvant and salvage irradiation in $34.1 \%$ and $2.4 \%$ of the cases, whereas patients who did not receive neoadjuvant treatment had adjuvant and salvage radiotherapy in $35.9 \%$ and $7.6 \%$ of the cases ( $p=0.856$ and $p=0.278$ ).

Conclusion: Although neoadjuvant hormonal treatment improved local tumor control, it did not reduce the frequency of secondary radiotherapy significantly. Overall, and in the CT2 subgroup, there was a relative decrease in the number of adjuvant treatments compared to salvage treatments in neoadjuvant-treated patients.

Keywords: High-risk carcinoma, irradiation, neoadjuvant hormonal treatment, prostate cancer, radical prostatectomy
\end{abstract}

\section{Introduction}

The most common type of cancer in men is prostate cancer. Treatment for low- and intermediate-risk prostate tumors has been resolved; however, therapy for high-risk cancers has not been fully resolved yet. According to D'Amico's classification of prostate cancer, a high-risk tumor has a prostatespecific antigen (PSA) of $\geq 20 \mathrm{ng} / \mathrm{mL}$, a Gleason score of $\geq 8$, or a cT3 grade tumor. Following radical prostatectomy for high-risk prostate tumors, biochemical progression (BCP) occurs in $55-70 \%$ of cases $(1,2)$. In in $13 \%$ of these cases, distant metastasis occurs, and tumorrelated mortality occurs in $6 \%$.

Currently, radiation therapy, in conjunction with hormonal treatment and multimodal treatment, can be used to successfully treat high-risk prostate tumors $(1,2,3)$. After radical prostatectomy, adjuvant or salvage radiation can be administered as part of a multimodal treatment depending on the histology and postoperative PSA levels. In terms of definitive radiation therapy, randomized clinical trials have shown that hormonal treatment combined with irradiation - especially longterm hormonal treatment - improved oncological outcomes $(4,5)$. The role of neoadjuvant hormonal therapy (ADT) prior to radical prostatectomy remains unknown. According to the European Association of Urology guidelines, the routine use of neoadjuvant hormonal therapy is not recommended before radical surgery of high-risk prostate tumors. However, several studies have shown that neoadjuvant hormonal treatment improves local tumor control $(5,6,7,8,9)$. In multimodal 
treatments, the efficacy of neoadjuvant ADT in slowing tumor progression and thus improving tumor-specific survival rates has not been demonstrated $(6,10)$. The effect of neoadjuvant therapy on the frequency of adjuvant and salvage radiotherapy is still unknown.

In this study, we investigated the efficacy of neoadjuvant androgen deprivation hormonal treatment before radical prostatectomy in patients with high-risk prostate cancer, as well as the need, if any, for subsequent adjuvant and salvage radiation treatment. Several studies have looked into the potential benefits of neoadjuvant treatment on tumor progression, but it has not really been investigated for secondary treatments. Our retrospective study included a relatively large number of patients with a long-term follow-up period.

\section{Materials and Methods}

From January 1996 to January 2019, our institute treated 527 patients with radical prostatectomy for high-risk prostate cancer.

On all patients with high-risk cancer, imaging was performed to determine the local extension of the tumor. Previously, (computed tomography) CT was usually performed before moving on to magnetic resonance imaging (MRI). There were 243 CT scans, 247 pelvic MRI scans, and 46 endorectal MRI scans. In 394 cases, imaging showed clinically localized cancer, while in 133 cases, imaging revealed locally advanced prostate cancer. In these patients, imaging procedures such as bone scintigraphy revealed no metastases.

Patients with high-risk prostate tumors were divided into two groups. Before radical prostatectomy, patients in group 1 $(n=139)$ received neoadjuvant androgen deprivation treatment. On the other hand, patients in group $2(n=388)$ did not receive any hormonal therapy prior to surgery. In group 1, the patients had a mean age of $64.5 \pm 6.2$ years and a mean PSA level of $32.5 \pm 24.5 \mathrm{ng} / \mathrm{mL}$. In group 2 , the mean age was $63.8 \pm 6.3$ years and the mean PSA level was $24.0 \pm 21.0 \mathrm{ng} / \mathrm{mL}$. The neoadjuvant hormonal treatment lasted between 3 and 6 months.

In our institute, we primarily used neoadjuvant hormonal treatment when the patient's PSA value was significantly higher or there was a significant extraprostatic tumor extension based on imaging. However, in some cases, the patient already begun hormonal treatment before being referred to our unit for radical prostatectomy.

The histological stage was determined retrospectively using the 2016 UICC TNM system. Following surgery, postoperative treatment was administered according to the current European Association of Urology protocol. During the follow-up period, PSA was measured every 3 months for the first 3 years, then every 6 months for another 5 years, and then annually after that. Imaging was performed on PSA elevation or following patient complaints. BCP was defined as an increase in PSA above 0.2 $\mathrm{ng} / \mathrm{mL}$ on at least two occasions.

\section{Statistical Analysis}

Statistical analyses were performed using the Student's t-test. A p-value of less than 0.05 was considered significant. We used Microsoft (MS) Excel and open-source R-programming packages. First, we visualized data distribution with histograms and boxplots using the "gplots" and "ggplot2" R-packages (R package version 3.0.3; available from https://CRAN.R-project. org/package=gplots) (11). Then, we illustrated the dichotomic clinical data using correlation plots by the "corrplot" package ( $R$ package "corrplot": Visualization of a Correlation Matrix-Version 0.84; available from https://github.com/taiyun/corrplot). To assess the statistical difference in frequencies, we calculated the relative risks in the two groups. We then checked to see if their quotient (RR) differed significantly from one another ( $p$-value). The relative risk and its significance were evaluated using MS Excel Pivotal tables or the "epitools" and "epiDisplay" R-packages (R package version 0.5-10.1; available from https:// CRAN.R-project.org/package=epitools). We used the Kendall correlation to estimate associations in clinical datasets.

The study was approved by the Ethical Committee of University of Debrecen (approval number: DE RKEB/IKEB: 5504-2020).

\section{Results}

Preoperative PSA levels were significantly higher in patients receiving neoadjuvant hormonal therapy $(p=0.0007)$. Based on the imaging, clinically locally advanced prostate cancer was detected in 41 cases (29.5\%) in group 1 and 92 cases (23.7\%) in group $2(p=0.194)$.

In group 1, histology showed locally advanced (pT3) tumors in 52 patients (37.4\%) and margin positivity in 26 patients (18.7\%) (Table 1).

The occurrence of pT3 stage and margin positivity was significantly lower in the subgroup receiving neoadjuvant treatment. We found no substantial changes in the pN1, BCP, or local recurrence subgroups.

In group 2, pT3 stage tumors were detected in 214 patients (55.2\%), with125 patients (32.2\%) having a positive surgical margin. Locally advanced prostate cancer and margin positivity were more common in the non-neoadjuvant hormonal treatment group $(p=0.00057$ and $p=0.0017)$. Lymph node positivity was similar in both groups $(p=0.72)$. The mean followup period was 50 months.

During the follow-up period, BCP was diagnosed in 27 patients (19.4\%) in group 1 and 54 patients (13.9\%) in group 2 $(p=0.130)$.

\section{Clinically organ-confined (cT2) tumors $(n=394)$ :}

In this subgroup, 98 patients received neoadjuvant hormonal treatment, while 296 patients did not. Among those patients, in the neoadjuvant hormone treatment group, histology showed 36 patients (36.7\%) having locally advanced (pT3) tumors, while 18 patients (18.3\%) had margin positivity. In the neoadjuvant hormonal treatment group, pT3 grade tumors were found in 154 cases (52\%), with 91 patients (30.7\%) having a positive surgical margin. Locally advanced prostate cancer and margin positivity were significantly more common in the nonneoadjuvant hormonal treatment group $(p=0.012$ and $p=0.02)$.

During the follow-up period, BCP was diagnosed in 19 patients (19.3\%) in the neoadjuvant hormone treatment group and in 41 patients $(13.8 \%)$ in the non-neoadjuvant group. There was no statistically significant difference between the two groups. 
Table 1. Changes in the frequencies of this clinical trial are shown as a result of radical prostatectomy after neoadjuvant pretreatment. To assess the statistical difference in frequencies, we calculated the relative risks in the two groups and then checked to see if their quotient (RR) was significantly different from one ( $p$-value). We also gave a $95 \%$ confidence interval for the risk ratio

\begin{tabular}{|c|c|c|c|c|c|c|}
\hline \multirow{2}{*}{ Signs } & \multirow{2}{*}{ Neoadjuvant } & \multirow{2}{*}{ Control } & \multirow{2}{*}{ p-value } & \multirow{2}{*}{ Risk ratio } & \multicolumn{2}{|c|}{ 95\% confidence interval of the RR } \\
\hline & & & & & lower & upper \\
\hline pT3 (n) & 52 & 214 & 0.0003 & 0.68 & 0.54 & 0.86 \\
\hline $\mathrm{pN} 1(\mathrm{n})$ & 14 & 35 & 0.7048 & 1.12 & 0.62 & 2.01 \\
\hline pN1 (\%) & 10.1 & 9 & & & & \\
\hline $\mathrm{BCR}(\mathrm{n})$ & 27 & 54 & 0.1301 & 1.40 & 0.92 & 2.12 \\
\hline BCR (\%) & 19.4 & 13.9 & & & & \\
\hline Local recurrence $(n)$ & 4 & 11 & 0.9482 & 1.02 & 0.33 & 3.14 \\
\hline Local recurrence (\%) & 2.9 & 2.8 & & & & \\
\hline
\end{tabular}

\section{Clinically locally advanced (cT3) tumors $(n=133)$ :}

In this subgroup, 41 patients received neoadjuvant androgen deprivation therapy, while 92 patients did not. In the neoadjuvant hormonal treatment group, histological examination revealed locally advanced (pT3) cancers in 16 cases (39.0\%) and margin positivity in 8 patients (19.5\%). In the non-neoadjuvant treatment group, pT3 grade tumors were found in 60 patients (65.2\%), while 34 patients (36.9\%) had positive surgical margin. Locally advanced prostate cancer and margin positivity were significantly more common in the non-neoadjuvant hormonal treatment group $(\mathrm{p}=0.008$ and $\mathrm{p}=0.05)$.

During the follow-up period, BCP was observed in 8 patients (19.5\%) in group 1 and in 13 patients (14.1\%) in group 2. There was no statistically significant difference between the two groups.

\section{Adjuvant and Salvage Treatment}

Adjuvant and salvage irradiation were performed in $22.3 \%$ and $5.8 \%$ of patients in group 1, respectively, and in $25.0 \%$ and $5.9 \%$ of patients in group 2 , respectively $(p=0.518$ and $\mathrm{p}=0.940$ ) (Table 2).

Using the risk ratio, we could evaluate relative changes in the numbers of treated patients. Overall, and in the CT2 subgroup, we observed a relative decrease in the number of adjuvant treatments compared to salvage treatments in the cases of neoadjuvanttreated patients. In the CT3 subgroup, however, the opposite trend was observed. None of the changes were statistically significant in terms of the patient numbers we examined.

Figure 1 depicts the distribution of secondary treatments.

Figure 2 depicts the pairwise correlations between neoadjuvant hormone-treated and non-hormone-treated parameters of patients. These analyses show the types of associations that were observed between the various clinical signs of disease monitored, patient outcome, and the therapeutical path taken in neoadjuvant-treated and non-treated cases of the study. Moreover, the linear relationship between the linked parameters is represented by black dots. The white dots represent the inverse relationship, and a lack of dots indicates a nonsignificant association. The size of the dots reflects the extent of the association. In neoadjuvant-pretreated cases, the adjuvant-ADT and adjuvant-irradiation treatment raws were better correlating with the higher preoperative PSA level, the more positive clinical imaging ( $\mathrm{CT} 3$ ) sign, the larger preoperative Gleason score, the pT3 and N1, and the margin positivity columns. These correlations indicate the presence of signs, which resulted in the neoadjuvant pretreatment. It is an inherent bias in our study that can be explained by an unintentional exclusion of less severe cases from the non-hormonal-treated group prior to surgery.

In the preoperative non-hormonal-treated cases, margin positivity had a stronger linear correlation with adjuvant-ADT and adjuvant-irradiation therapy than in hormone-treated cases. Salvage-ADT and salvage-irradiation therapies were found to be more strongly correlated with $\mathrm{BCP}$, local recurrence, metastasis, and increased mortality. The lack of hormonal pretreatment increases the likelihood of reaching more clinically severe outcomes.

\section{Discussion}

The most common malignant disease in men is prostate cancer, with $30 \%$ of cases being high risk. At present, there are conflicting views on the best way to treat high-risk prostate carcinomas $(12,13,14)$.

Neoadjuvant treatments used prior to surgery serve two purposes: one is to provide local tumor control and the other is to provide systemic management of microscopic metastases. The initially locally advanced tumor may become operable, with a higher chance of eliminating removable tumors due to improved local tumor control.

In high-risk prostate tumors, neoadjuvant treatment prior to radical prostatectomy may also help provide better tumor control, decrease local or locally advanced tumors, and eradicate microscopic metastases. 


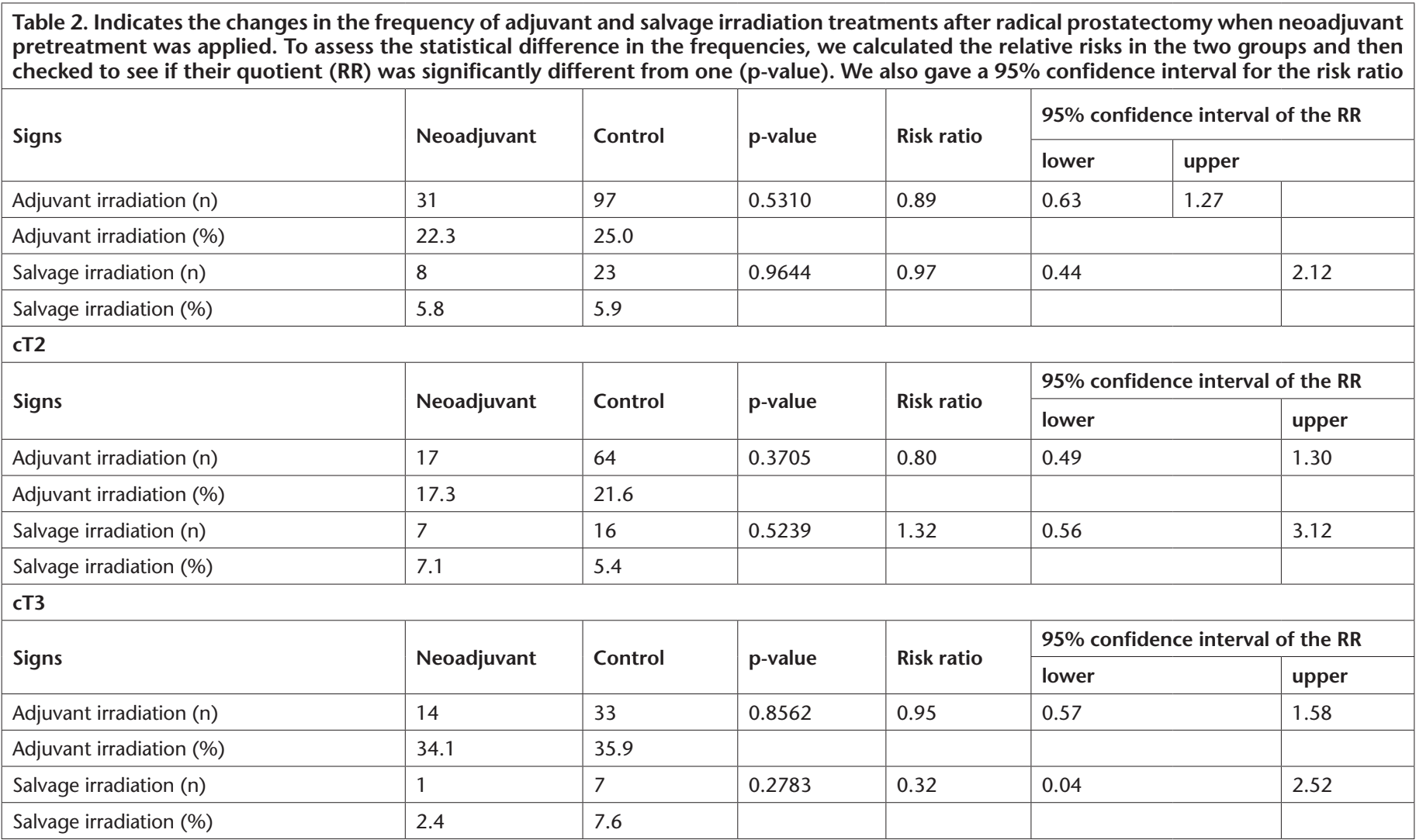
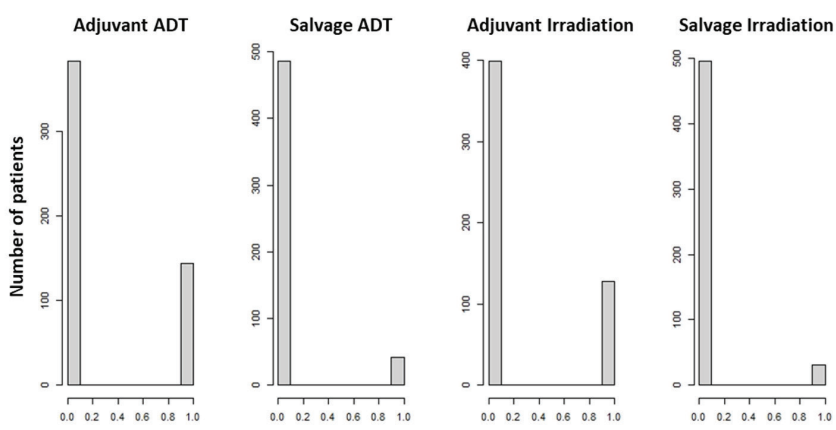

Adjuvant ADT
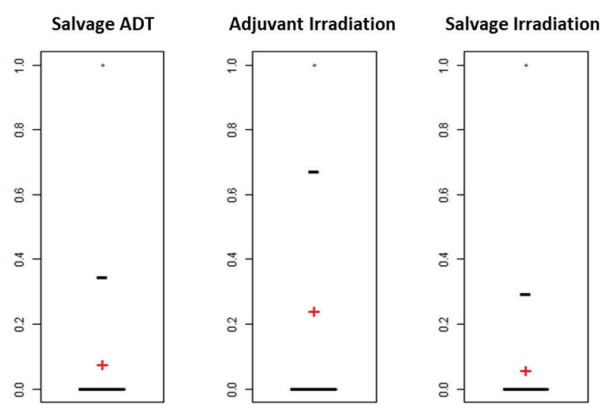

Figure 1. The histograms depict the distribution of the posttreatment data

The upper histograms depict the distribution, while the bottom boxplots show the median (horizontal thick black line), minimum (lowest horizontal line), maximum (top dot), and first and third quartiles (regions shaded to gray) of the posttreatment dataset. Red "+" is the mean, and black "-" is the standard deviation from the mean into the boxplot's positive direction
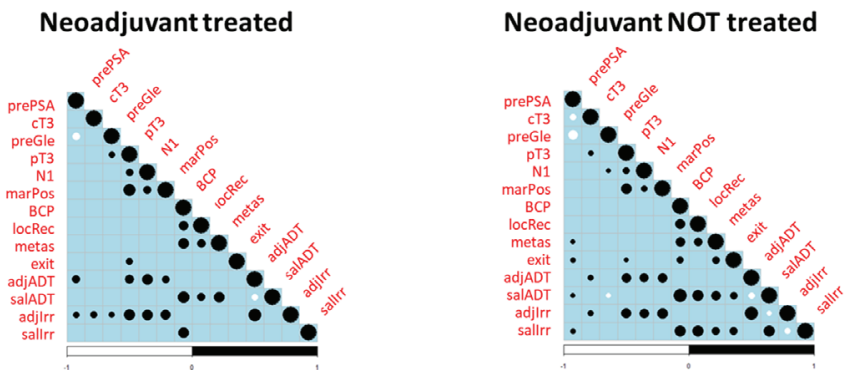

Figure 2. This figure illustrates the pairwise correlations between clinical parameters in the neoadjuvant hormone-treated and non-treated groups

The relationship between the data was calculated using the Kendall correlation coefficients. The size of the black and white dots is proportional to the value of the coefficients. According to the scale below the triangles, the black dot indicates a linear relationship, while the white dot indicates the reverse relationship between the parameters. No dot was included in the table if the deviation of the correlation coefficient's value from 0 was not significant $(p>0.05)$

McKay et al. (7), in their meta-analysis of ten clinical trials, reported a significant reduction in pathological T-stage prostate tumors after neoadjuvant hormonal treatment, an increase in the proportion of tumors localized to the prostate, a decrease in margin-positive cases, and a decrease in lymph node metastases. In several of their studies, Hsu et al. (15) reported that the significant prognostic factors after radical prostatectomy in CT3 patients are tumor differentiation, marginal positivity, and lymph node positivity. These are the most critical factors determining the 10-year BCP-free survival, clinical progression-free survival, tumor-specific survival, and overall survival (16). 
Hu et al. (17), in their meta-analysis of neo-adjuvant hormonal treatments for non-metastatic prostate tumors, found that the time to BCP and overall survival was significantly increased. In their multicenter study of high-risk prostate cancer patients, Tosco et al. (18) also found that neoadjuvant hormonal therapy significantly reduced tumor-induced mortality.

However, most clinical trials of neoadjuvant hormonal therapy before radical prostatectomy found that BCP and overall survival did not improve $(7,19)$.

Although local tumor control was better in our study, there was no significant difference in BCP and survival rates between the two groups.

In our study, we examined the benefits of neoadjuvant hormonal therapy before radical prostatectomy in patients with high-risk prostate cancer, both in terms of oncological outcomes and possible secondary treatment regimens. Several studies have been conducted to investigate the efficacy of neoadjuvant treatment on tumor progression. However, it has not been thoroughly investigated how the frequency of adjuvant and salvage treatments could be adjusted following neoadjuvant treatment. When considering neoadjuvant hormonal treatment before surgery, it is critical to remember that neoadjuvant treatment can have an impact on the overall therapy administered. Adjuvant and salvage therapies administered following radical prostatectomy are essential for improving BCPfree survival, tumor-free survival, and quality of life.

If the frequency of the required adjuvant and salvage treatments was reduced as a result of neoadjuvant therapy, with the same oncological outcome, patients' quality of life would be significantly improved. It is well known that complications are common following adjuvant and salvage radiotherapy.

After adjuvant irradiation, the incidence of early and late complications ranges from 15 to $35 \%$ and from 2 to $8 \%$, respectively (20).

However, in our study, there was no significant difference in the frequency of adjuvant and salvage treatments between the two groups. Our data showed that adjuvant radiotherapy was less frequently required in the neoadjuvant hormonal treatment group, although preoperative PSA was significantly higher.

\section{Study Limitations}

Our study had some limitations. For starters, it was a retrospective rather than a prospective study. Second, we found no significant benefit of neoadjuvant hormonal therapy in reducing the need for a secondary treatment, and we discovered that only the hormonal pretreatment had a tendency to reduce the number of adjuvant treatments (especially in cT2 subgroup).

\section{Conclusion}

Our results showed that neoadjuvant hormonal treatment provided significantly better local tumor control in the cases of radical prostatectomies for locally advanced high-risk prostate tumors. Neoadjuvant hormonal therapy had no effect on tumor progression and did not reduce the number of adjuvant and salvage treatments required. However, the correlation analysis showed that, overall and in the cT2 subgroup, a relative decrease in the number of adjuvant treatments was observed in patients who received neoadjuvant treatment compared to those who received salvage treatments. Although the benefits of the neoadjuvant hormonal treatment for adjuvant irradiation did not reach statistical significance in patients with high-risk prostate cancer, there was strong evidence of a benefit from neoadjuvant treatment.

\section{Acknowledgements}

Publication: The results of the study were not published in full or in part in form of abstracts.

Contribution: There is not any contributors who may not be listed as authors.

Conflict of Interest: No conflict of interest was declared by the authors.

Financial Disclosure: The authors declared that this study received no financial support.

\section{Ethics}

Ethics Committee Approval: The study was approved by the Ethical Committee of University of Debrecen (approval number: DE RKEB/IKEB: 5504-2020).

Informed Consent: Retrospective study.

Peer-review: Externally peer-reviewed.

\section{Authorship Contributions}

Critical Review: T.F., Concept: C.B., Design: C.B., Data Collection or Processing: C.B., J.D., Analysis or Interpretation: Z.B., Literature Search: Z.B., Writing: C.B., J.D., B.T.

\section{References}

1. Loeb S, Schaeffer EM, Trock B], et al. What are the outcomes of radical prostatectomy for high-risk prostate cancer? Urology 2010;76:710-714.

2. Yossepowitch O, Eggener SE, Serio AM, et al. Secondary therapy, metastatic progression, and cancer-specific mortality in men with clinically high-risk prostate cancer treated with radical prostatectomy. Eur Urol 2008;53:950-959.

4. Bolla M, de Reijke TM, Van Tienhoven G, et al. Duration of androgen suppression in the treatment of prostate cancer. N Engl J Med 2009;360:2516-2527.

4. Horwitz EM, Bae K, Hanks GE, et al. Ten-year follow-up of radiation therapy oncology group protocol 92-02: a phase III trial of the duration of elective androgen deprivation in locally advanced prostate cancer. J Clin Oncol 2008;26:2497-2504.

5. Zapatero A, Guerrero A, Maldonado X, et al. High-dose radiotherapy with short-term or long-term androgen deprivation in localised prostate cancer (DART01/05 GICOR): a randomised, controlled, phase 3 trial. Lancet Oncol 2015;16:320-327.

6. Klotz LH, Goldenberg SL, Jewett MA, et al. Long-term follow-up of a randomized trial of 0 versus 3 months of neoadjuvant androgen ablation before radical prostatectomy. J Urol 2003;170:791-794.

7. McKay RR, Choueiri TK, Taplin ME. Rationale for and review of neoadjuvant therapy prior to radical prostatectomy for patients with high-risk prostate cancer. Drugs 2013;73:1417-1430.

8. McKay RR, Montgomery B, Xie W, et al. Post prostatectomy outcomes of patients with high-risk prostate cancer treated with neoadjuvant androgen blockade. Prostate Cancer Prostatic Dis 2018;21:364-372. 
9. Schulman CC, Debruyne FM, Forster G, et al. 4-Year follow-up results of a European prospective randomized study on neoadjuvant hormonal therapy prior to radical prostatectomy in T2-3NOM0 prostate cancer. European Study Group on Neoadjuvant Treatment of Prostate Cancer. Eur Urol 2000;38:706-713.

10. Berglund RK, Tangen CM, Powell IJ, et al. Ten-year follow-up of neoadjuvant therapy with goserelin acetate and flutamide before radical prostatectomy for clinical T3 and T4 prostate cancer: update on Southwest Oncology Group Study 9109. Urology 2012;79:633-637.

11. Wickham H. ggplot2: elegant graphics for data analysis. New York: Springer-Verlag; 2016.

12. Baker $C B$, McDonald AM, Yang ES, et al. Pelvic radiotherapy versus radical prostatectomy with limited lymph node sampling for high-grade prostate adenocarcinoma. Prostate Cancer 2016;2016:2674954. doi: 10.1155/2016/2674954.

13. Kishan AU, Shaikh T, Wang PC, et al. Clinical outcomes for patients with gleason score 9-10 prostate adenocarcinoma treated with radiotherapy or radical prostatectomy: a multi-institutional comparative analysis. Eur Urol 2017;71:766-773.

14. Matulay JT, DeCastro GJ. Radical prostatectomy for high-risk localized or node-positive prostate cancer: removing the primary. Curr Urol Rep 2017;18:53.
15. Hsu CY, Joniau S, Roskams $\mathrm{T}$, et al. Comparing reults after surgery in patients with clinical unilateral T3a, prostate cancer treated with or without neoadjuvant androgen deprivation therapy. BJU Int 2006;99:311-314.

16. Hsu $\mathrm{CY}$, Joniau $\mathrm{S}$, Oyen R, et al. Outcome of surgery for clinical clinical unilateral T3a: a single institution experience. Eur Urol 2007;51:121-129.

17. Hu J, Xu H, Zhu W, et al. Neo-adjuvant hormone therapy for nonmetastatic prostate cancer: a systematic review and meta-analysis of 5,194 patients. World J Surg Oncol 2015;22;13:73.

18. Tosco L, Laenen A, Briganti A, et al. The survival impact of neoadjuvant hormonal therapy before radical prostatectomy for treatment of highrisk prostate cancer. Prostate Cancer Prostatic Dis 2017;20:407-412.

19. Gandaglia G, Sun M, Trinh QD, et al. Survival benefit of definitive therapy in patients with clinically advanced prostate cancer: estimations of the number needed to treat based on competing-risks analysis. BJU Int 2014;114:E62-E69. doi: 10.1111/bju.12645.

20. Gandaglia G, Briganti A, Clarke N, et al. Adjuvant and Salvage Radiotherapy after Radical Prostatectomy in Prostate Cancer Patients. Eur Urol 2017;72:689-709. 